\title{
Emploi des temps et cohérence textuelle dans
}

L'Occupation des sols

Catherine Fuchs, Pierre Le Goffic et Mathieu Maillard

\section{(2) OpenEdition \\ Journals}

Édition électronique

URL : http://journals.openedition.org/rsl/794

DOI : $10.4000 /$ rsl. 794

ISSN : 2271-6246

Éditeur

Éditions Rue d'Ulm

Référence électronique

Catherine Fuchs, Pierre Le Goffic et Mathieu Maillard, « Emploi des temps et cohérence textuelle dans L'Occupation des sols », Revue Sciences/Lettres [En ligne], 3 | 2015, mis en ligne le 08 décembre 2014, consulté le 19 avril 2019. URL : http://journals.openedition.org/rsl/794 ; DOI : 10.4000/rsl.794

Ce document a été généré automatiquement le 19 avril 2019

(c) Revue Sciences/Lettres 


\title{
Emploi des temps et cohérence textuelle dans L'Occupation des sols
}

\author{
Catherine Fuchs, Pierre Le Goffic et Mathieu Maillard
}

1 La présente contribution est consacrée à l'emploi des temps dans L'Occupation des sols. À travers des représentations d'événements et de situations, les temps verbaux contribuent, comme on sait, à la cohérence du récit. Dans une narration classique, comme par exemple Le Petit Fût de Maupassant, le récit se déroule linéairement et les événements successifs s'enchaînent de façon suivie. Dans le texte d'Echenoz, au contraire, coupes discontinues, ruptures de plans, raccourcis, avancées et retours en arrière donnent, pour ainsi dire, une vision stroboscopique de l'histoire, laissant au lecteur le soin de combler les « trous » du récit et l'obligeant à tisser lui-même la trame narrative, afin d'en restituer la cohérence globale.

2 Dans une première partie, nous examinerons le déroulement du récit. Dans une seconde partie, nous nous arrêterons sur quelques emplois remarquables des temps.

\section{Le déroulement du récit}

\subsection{Les époques temporelles au fil du texte: chronologie des faits et emploi des temps}

Constitué de quinze paragraphes successifs, le récit peut, très sommairement, être divisé en trois grandes époques (entremêlées de plusieurs flash-backs).

\subsubsection{Première époque : exposition}

4 Les paragraphes 1 à 5 , presque uniquement à l'imparfait (vingt-huit occurrences) et au plus-que-parfait (cinq occurrences), constituent l'exposition d'une situation: la vie commune d'un père (Fabre) et de son fils (Paul) après un incendie. Exposition accompagnée de divers flash-backs: quatre des occurrences de plus-que-parfait 
concernent l'image de la mère (Sylvie), qu'un peintre (Flers) avait représentée, faisant la publicité d'un parfum, sur le mur extérieur d'un immeuble (l'immeuble Wagner).

5 Imparfaits descriptifs d'états de choses précis alternent avec des imparfaits itératifs référant aux propos et gestes habituels du père dans l'appartement ainsi qu'à la visite (bi)hebdomadaire du père et du fils à l'image de la mère. Dans cette partie ne figure pratiquement qu'un seul véritable événement, marqué par le premier des trois passés simples (trouva), qui réfère à la réinstallation des personnages dans un nouveau logement et marque un changement d'état effectif, instaurant à ce titre un début de narration.

\subsubsection{Deuxième époque : péripétie}

6 Ce n'est qu'à partir du paragraphe 6 que commence véritablement la narration. Située dans une époque ultérieure (plus tard) indéterminée mais clairement distante de la précédente (le fils, devenu autonome, ne vit plus avec son père), elle s'étend sur un intervalle de temps lui aussi indéterminé. Y sont relatées les transformations successives qui affectent les deux flancs de l'immeuble Wagner, au fil des visites du fils. D'un côté, un bâtiment insalubre remplacé par une construction moderne; de l'autre, un espace vert d'abord laissé à l'abandon puis livré à un promoteur - en sorte que le nouvel immeuble qui s'y construit masque progressivement l'image de la mère.

7 Cette partie, qui va du début du paragraphe 6 jusqu'au milieu du paragraphe 10 (la charnière avec la troisième partie étant marquée par la phrase Paul cessa de le visiter lorsque la robe entière eut été murée), se caractérise par une sorte d'instabilité du récit dont témoigne la diversité des temps employés. Si l'équilibre quantitatif entre imparfait (seize occurrences) et passé simple (seize occurrences) pourrait laisser penser à la mise en place d'une narration classique, plusieurs facteurs viennent pourtant en troubler le fil. En premier lieu, trois occurrences de conditionnel prospectif ont pour effet de projeter les procès et états de choses, depuis l'époque passée en question vers un futur indéterminé, sur le mode d'une programmation voire d'une fatalité annoncée : «(de par le destin), tel devait être le cas, cela allait nécessairement advenir ». Par ailleurs, la présence, au premier abord très déroutante, de quinze occurrences de présent (qui se donnent à lire comme génériques) et de plusieurs participes (qui se présentent eux aussi comme hors successivité et hors actualisation dans la situation particulière) semble arrêter le fil du récit et inciter le lecteur à abandonner l'histoire au profit d'une sorte de réflexion générale. Mais ces excursions génériques sont elles-mêmes clôturées par des reprises du récit, en sorte qu'il revient au lecteur de les réintégrer dans la trame narrative.

\subsubsection{Troisième époque : dénouement ?}

8 Elle commence à la deuxième partie du paragraphe 10 (Des semaines passèrent [...]) et se termine à la fin du quinzième et dernier paragraphe. La césure temporelle qui scinde le dixième paragraphe et marque le tournant narratif du texte s'explique par le fait que l'unité profonde de ce paragraphe réside, non pas dans la chronologie des événements, mais bien plutôt dans la description de la transformation qualitative du lieu décrit : l' espace vert [...] qui formait un gazon subsidiaire aux pieds de Sylvie (comme indiqué au §6) a été remplacé par un édifice c'est-à-dire, aux yeux du fils (et du père), un sépulcre au lieu d'une effigie de Sylvie. 
9 La troisième époque présente moins de difficultés dans la restitution du fil narratif. Elle se situe des semaines (combien ?) après la fin des visites du fils au chantier. L'histoire s'accélère ; tout se passe en quelques jours : retour accidentel du fils et retrouvailles avec le père installé dans le nouvel immeuble (microscène), description du nouveau logement $\mathrm{du}$ père, flash-back sur l'emménagement du père, suite des retrouvailles, retour du fils pour le week-end, récit de leurs échanges, enfin déjeuner puis attaque du grattage du mur (pour retrouver l'image de la mère). Les alternances entre imparfaits descriptifs et passés simples aoristiques paraissent plus classiques, du moins au premier abord. Restent toutefois le problème d'interprétation des trois occurrences de conditionnel et celui des douze occurrences de présent (dont un passé composé valant accompli présent) dans le dernier paragraphe. C'est précisément sur ces présents d'interprétation incertaine (procès dynamiques en situation? activités itératives ou génériques ?) que se termine le texte.

\subsubsection{Schéma d'ensemble}

10 À l'issue de cette brève esquisse des trois grandes étapes du récit qu'il est possible de reconstruire à partir du texte, on peut récapituler la chronologie d'ensemble (y compris les autres époques évoquées par des flash-backs) dans le schéma suivant.

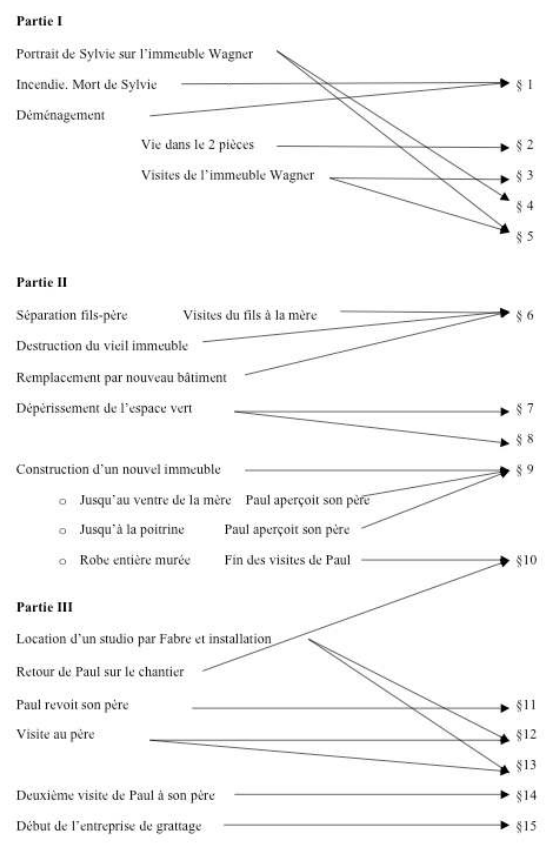

11 Cette reconstitution chronologique rend visibles deux caractéristiques principales du récit, considéré sous l'angle de la temporalité.

D’abord, la disparité des intervalles liés à chacune des trois grandes périodes. Pour la première période, il s'agit d'un intervalle (indéterminé) relativement long. On peut supposer en effet que la vie commune du père et du fils après l'incendie a duré plusieurs années jusqu'à la fin de l'adolescence du fils. Au sein de cette période, leur vie commune est décrite à l'échelle de la journée en ce qui concerne les activités d'intérieur (le soir après le dîner; chambre à coucher), et à celle de la semaine s'agissant des visites à l'image de la 
mère (le dimanche et certains jeudis). Pour la seconde période, l'intervalle (également non spécifié) est vraisemblablement plus court. La destruction/reconstruction de l'immeuble de gauche, le dépérissement de l'espace vert et l'édification du nouvel immeuble n'ont guère pris moins d'années et se mesurent peut-être même en mois. Enfin, pour la troisième période, il s'agit d'un intervalle très court et davantage déterminé. Le retour du fils, ses retrouvailles avec son père dans le nouvel appartement et sa deuxième visite le week-end suivant, semblent en effet se dérouler sur une seule semaine. La construction du récit obéit donc clairement à une logique d'accélération temporelle: d'abord une phase d'états et d'activités itératives (en années), puis une autre de transformations progressives (en mois), et enfin une dernière phase d'événements ponctuels (en jours), qui culmine sur une sorte d'" arrêt sur image ».

Seconde caractéristique, la coexistence de circonstants temporels et de «trous » dans la chronologie. On sait (cf. flash-back des $§ 4$ et 5) que Flers avait peint l'image de Sylvie trois ans avant la naissance de Paul; mais on ne sait pas quand était né Paul ni quand avait eu lieu l'incendie (et donc quel âge avait Paul au début du récit). On sait que le père et le fils déménagent dans le deux pièces tout de suite après l'incendie (trop vite); mais on ne sait pas combien de temps ils y restent. On sait qu'après la séparation (non datée) du père et $\mathrm{du}$ fils (plus tard), ce dernier revient voir l'image de sa mère (deux ou trois fois par mois, parfois plus), qu'il aperçoit son père sur le chantier par deux fois (une fois [...] et une autre fois), et qu'il cesse de s'y rendre lorsque la robe de Sylvie est entièrement murée. Enfin, on sait que des semaines après, Paul revient accidentellement sur le chantier, retrouve son père et monte le voir (pendant vingt minutes) dans le nouvel appartement que celui-ci vient de louer (cf. flash-backs des $\$ 12$ et 13); puis qu'il y revient le samedi soir suivant et reste le dimanche. Toutes ces indications livrent des repères relatifs et assez indéterminés, ne permettant ni datation objective ni enchaînement complet et précis. Le lecteur n'est donc en mesure de reconstruire que des séquences temporelles partielles et locales.

\subsection{La distribution des temps dans le texte}

14 Nous avons choisi de relever toutes les formes verbales du texte et de les surligner en fonction des temps employés. Chaque temps donne donc lieu à un coloriage spécifique, valant également pour les temps composés correspondants. Par exemple ici, six couleurs ont été nécessaires ; nous avons (arbitrairement) retenu les suivantes :

- jaune pour les imparfaits et plus-que-parfaits ;

- rose violacé pour les passés simples et passés antérieurs;

- gris pour les présents et passés composés ;

- turquoise pour les subjonctifs présents ou imparfaits ;

- marron clair pour les impératifs ;

- et vert brillant pour les conditionnels.

L'encadré ci-dessous donne un échantillon de l'emploi des différents temps dans les paragraphes 1,2 et 15. 
\$1 Comme tout avait brûlé - la mère, les meubles et les photographies de la mère -, pour Fabre et le fils Paul c'était tout de suite beaucoup d'ouvrage : toute cette cendre et ce deuil, déménager, courir se refaire dans les grandes surfaces. Fabre trouva trop vite quelque chose de moins vaste, deux pièces aux fonctions permutables sous une cheminée de brique dont l'ombre donnait l'heure, et qui avaient ceci de bien d'être assez proches du quai de Valmy.

\$2 Le soir après le dîner, Fabre parlait à Paul de sa mère, sa mère à lui Paul, parfois dès le dîner. Comme on ne possédait plus de représentation de Sylvie Fabre, il s'épuisait à vouloir la décrire toujours plus exactement: au milieu de la cuisine naquirent des hologrammes que dégonflait la moindre imprécision. Ça ne se rend pas, soupirait Fabre en posant une main sur sa tête, sur ses yeux, et le découragement l'endormait. Souvent ce fut à Paul de déplier le canapé convertible, transformant les choses en chambre à coucher.

\$15 Le soleil en effet balaierait tout le studio, comme un projecteur de poursuite dans un music-hall frontalier. C'était dimanche, dehors les rumeurs étouffées protestaient à peine, parvenant presque à ce qu'on les regrettât. Ainsi que tous les jours chômés, les heures des repas tendraient à se glisser les unes sur les autres, on s'entendit pour quatorze heures - ensuite on s'y met. Un soleil comme celui-ci, développa le père de Paul, donne véritablement envie de foutre le camp. Ils s'exprimèrent également peu sur la difficulté de leur tâche qui requerrait, c'est vrai, de la patience et du muscle, puis des scrupules d'égyptologue en dernier lieu. Fabre avait détaillé toutes les étapes du processus dans une annexe agrafée aux plans. Ils mangèrent donc vers quatorze heures mais sans grand appétit, leurs mâchoires broyaient la durée, la mastication n'était qu'horlogère. D'un tel compte à rebours on peut, avant terme, convoquer à son gré le zéro. Alors autant s'y mettre, autant gratter tout de suite, pas besoin de se changer, on a revêtu dès le matin ces larges tenues blanches pailletées de vieille peinture, on gratte et des stratus de plâtre se suspendent au soleil, piquetant les fronts, les cafés oubliés. On gratte, on gratte et puis très vite on respire mal, on sue, il commence à faire terriblement chaud.

Ce coloriage permet de prendre immédiatement conscience d'un certain nombre de caractéristiques du texte. On remarque ainsi :

- la prédominance quasi exclusive des jaunes (imparfaits et plus-que-parfaits descriptifs) dans les paragraphes 1 à 5 , puis à nouveau dans les paragraphes 8,12 ;

- l'irruption des roses violacés (passés simples, aoristes de récit), alternant avec les jaunes, dans le paragraphe 6 ; alternance qui se retrouve dans les paragraphes 9 à 11,13 et 15 ;

- l'irruption des gris (présents de commentaire général) dans les paragraphes 7 et $9 ;$ puis la réapparition de gris (présents sans doute souvent aoristiques) dans le paragraphe 15;

- la présence épisodique de certaines couleurs: un turquoise (subjonctif dans une subordonnée) aux paragraphes $6,8,10$ et 15 ; un marron (impératif signalant un discours direct) aux paragraphes 5, 11, 14; et enfin, un ou plusieurs vert(s) brillant(s) (conditionnel(s) prospectifs) aux paragraphes 6,8 et 15 .

Chacun de ces emplois appelle une analyse précise. Mais la visualisation d'ensemble donne déjà un aperçu quantitatif des grandes tendances, qui livre quelques clés concernant la structuration du texte en grandes périodes temporelles. Les indications données par la partie 1 du schéma pourront ainsi être croisées avec celles résultant des parties II et III de l'étape suivante, visant à aller plus avant dans la restitution de la chronologie des faits.

Toutefois, la difficulté à restituer le fil narratif dans une perspective temporelle tient moins à ces non-dits et à ces «trous " chronologiques qu'à l'instabilité interprétative induite par certains emplois déroutants des temps verbaux, comme nous allons le voir à présent. Quatre points retiendront notre attention: l'usage de certains imparfaits et passés simples, l'emploi des participes présents, le rôle des présents et celui des conditionnels. 


\section{Des imparfaits et des passés simples « étranges »}

19 L'étrangeté de certains emplois de ces deux temps se signale, d'une part dans les mises en séquence des temps, d'autre part dans le choix des lexèmes verbaux.

\subsection{Les mises en séquence des temps}

Comme il a été dit plus haut, la troisième partie du texte présente moins de difficultés dans la restitution du fil narratif que les deux premières. La mise en séquence des temps imparfait et passé simple (tant en alternance qu'en répétition) y semble relativement classique, à première vue. Pourtant, certains facteurs contribuent, comme nous allons le voir, à en déstabiliser l'interprétation.

\subsubsection{Les alternances imparfait / passé simple}

Dans leur emploi classique au sein d'un récit, l'imparfait et le passé simple alternent pour marquer, respectivement, un état de choses situé en arrière-plan et un événement se détachant sur ce fond; exemple : Tout était calme, soudain une sirène mugit. Ces deux effets prototypiques procèdent de la valeur linguistique propre à chacun des deux temps: valeur de procès en cours d'accomplissement pour l'imparfait, valeur d'aoriste pour le passé simple.

Le texte d'Echenoz comporte, dans sa troisième et dernière partie, des alternances d'imparfaits et de passés simples conformes à ce schéma classique, du moins en apparence.

23 Ainsi, au paragraphe 11, la séquence suivante: [...] une femme qui venait sur le trottoir s'arrêta derrière lui, leva les yeux au ciel et cria Fabre. Paul [...] se tourna vers celle qui criait Fabre Fabre encore [...]. La voix énervante tomba du ciel [...]. Cette séquence pourrait passer pour un récit tout à fait canonique. Mais au sein du paragraphe où elle apparaît, plusieurs autres inscriptions temporelles viennent en déstabiliser l'apparente normalité.

D'abord, la séquence est précédée, au tout début du paragraphe, par un imparfait et un participe qui, d'entrée de jeu, orientent vers une description des lieux, et non pas vers un récit d'événements : [...] un terre-plein meuble prédisait le retour de la végétation trahie. Paul considérant cela, [...].

Ensuite, trois occurrences de présent font interférer dans le récit des éléments adventices, plus ou moins difficiles à intégrer dans la trame: (Paul), dont c'est quand même le nom, [...] ; (celle qui criait Fabre Fabre encore,) j’ai du lait ; (La voix énervante tomba du ciel [....]) : tu simules, Jacqueline.

Enfin, le paragraphe se termine de façon énigmatique : La femme s'éloignait dépeint un procès en cours, comme suspendu, là où l'on attendrait un aoriste (s'éloigna) qui clôturerait la séquence narrative ; on ne sait pas qui c'était introduit un commentaire "off " sans prise en charge explicite; et l'impératif Monte, Paul condense à lui seul tout le processus interprétatif qui doit conduire le lecteur à attribuer ce propos au père (la voix énervante) et à comprendre que celui-ci occupe un logement tout en haut de l'immeuble nouvellement construit. 

des plus-que-parfaits (deux occurrences). Le moment d'observation y est donc stable, d'autant plus que les deux plus-que-parfaits s'interprètent comme des états résultants participant de la description de l'appartement, plutôt que comme des retours en arrière événementiels : Des revers avaient dû sévir [...] ; Il avait fait les vitres [...]. à la source des effets prototypiques induits, dans une narration classique, par une succession d'imparfaits ou par une succession de passés simples. Dans le premier cas, une accumulation de tableaux descriptifs sans avancée dans le temps; exemple : Il faisait beau, la mer était calme, le soleil brillait. Dans le second, une suite de procès qui se succèdent temporellement; exemple : Il prit son chapeau, mit ses gants et sortit.

Le texte d'Echenoz comporte lui aussi, dans sa troisième partie, de telles successions ; leur interprétation exige toutefois un travail plus complexe que dans les cas classiques.

\section{(a) Les successions d'imparfaits}

C'est à un autre type d'instabilité que le lecteur se trouve confronté face à l'alternance dimparfaits et de passés simples du paragraphe 13. À la fin du paragraphe précédent, le repère temporel des événements est le moment (appelons-le $\mathrm{T}$ ) où Paul, étant monté voir un plus-que-parfait marquant un retour en arrière, c'est-à-dire référant le procès décrit à une époque antérieure à $\mathrm{T}$ : Fabre s'était présenté le premier au bureau de location [...].

parfait, permettant ainsi de maintenir $\mathrm{T}$ comme ancrage temporel de base : par exemple franchement d'emménager tout de suite [...]. Cette suite pourrait laisser penser que l'événement ainsi relaté se situe en $\mathrm{T}$; or le contenu lexical indique qu'il n'en est rien, ce qui oblige le lecteur à effectuer une bascule de l'ancrage temporel, et à recalculer celui-ci comme antérieur à $\mathrm{T}$.

premier peut encore s'interpréter comme décrivant les lieux au moment (antérieur à T) où le père avait loué le studio : [...] emménager [...] dans un studio situé sous les yeux de Sylvie qui étaient deux lampes sourdes derrière le mur de droite. Mais le second décrit en fait un état les deux passés simples suivants, qui renvoient bien au moment de la visite de Paul : à son fils il démontra cela sur plans; Paul partit quand même au bout de vingt minutes.

pour effet d'en casser la successivité immédiate. Le premier, La voix de Fabre exposait une ] s'interprète comme une sorte de commentaire de à son fils il l'autruche, comme qualifiant l'effet sur Paul de la cause en question (non autrement spécifiée).

\subsubsection{Les successions d'imparfaits et de passés simples}


de vue associé à ces imparfaits. Si certains d'entre eux ont l'apparence de descriptions neutres et objectives, d'autres en revanche sont ambivalents, introduisant comme en surimpression des notations subjectives. Notations attribuables, pour les unes, à Paul en tant qu'observateur extérieur ne participant pas à l'état de choses décrit - ce dont témoigne la présence d'une modalité épistémique ou d'un verbe impliquant un sujet source de l'impression décrite : Des revers avaient dû sévir [...]; la proportion de bouteilles vides semblait normale, en revanche une multitude de chariots d'hypermarchés rivaux déconcertait; et pour les autres au contraire, à Fabre en tant que sujet expérientiel de l'état décrit : ne craignait pas l'eau froide.

Entre ces différents points de vue, le jeu est constant : Fabre se tenait bien vêtu peut aussi bien se comprendre comme une description objective que comme une appréciation du fils, ou même comme une auto-appréciation du père ; et la suite ne craignait pas l'eau froide comme un état subjectif du père confortant par là même l'auto-appréciation précédente, ou bien comme une inférence du fils confirmée par la suite ( « il ne craignait pas l'eau froide, (puisqu')il avait fait les vitres »). Ce type d'instabilité interprétative liée au point de vue associé à l'imparfait est récurrent dans le texte. On le retrouve, par exemple, s'agissant de la description de l'image (d'abord visible, puis cachée) de Sylvie : c'était une belle robe au décolleté profond, c'était une vraie mère (\$ 6), notation subjective qui contraste avec l'apparente objectivité de c'était un sépulcre (\$10).

\section{(b) Les successions de passés simples}

39 À l'inverse d'une telle accumulation d'imparfaits, le paragraphe 14 contient presque exclusivement des passés simples (sept occurrences) - exception faite d'un plus-queparfait (Le père avait fait quelques courses : état résultant), d'un imparfait (Fabre était un peu plus disert que Paul) ainsi que d'un impératif suivi d'un présent (Regarde un peu le soleil qu'on $a$ : citation au discours direct).

L'enchaînement des deux premiers passés simples induit l'effet classique d'une succession chronologique d'événements: Il rassembla des affaires et revint samedi soir. Tel n'est pas le 
cas, en revanche, des trois suivants: Nul ne raconta rien de ces dernières années, rien ne s'évoqua sous l'ampoule nue; on discourut juste de la nécessité, puis de la couleur d'un abat-jour. Cette séquence se signale en effet par la quasi-synonymie des trois procès évoqués ( raconter, évoquer, discourir) qui impose de n'y voir en définitive qu'un seul et même événement - ou plutôt non-événement, comme l'indiquent les marqueurs négatifs et indéterminés (nul, rien, on).

41 La continuation avec les deux verbes suivants, respectivement à l'imparfait et au passé simple est, à son tour, quelque peu déroutante : Fabre était un peu plus disert que Paul ; avant de s'endormir il se plaignit doucement [...]. Après le non-événement de parole auquel réfèrent les trois passés simples, on se serait en effet attendu à un aoriste pour renvoyer enfin à un événement de parole : Fabre fut un peu plus disert que Paul ; or c'est un imparfait décrivant un état que l'on trouve. Inversement, une fois accepté cet imparfait, l'on s'attendrait à une continuation sur le même registre : il se plaignait; or, là encore, le lecteur se trouve déstabilisé et contraint de changer le réglage de ses paramètres interprétatifs, puisqu'un circonstant (avant de s'endormir) vient au contraire annoncer le repérage temporel d'un événement.

\subsection{Le choix des lexèmes verbaux}

La valeur de procès en cours d'accomplissement de l'imparfait et la valeur d'aoriste du passé simple expliquent l'affinité naturelle de chacun de ces deux temps avec des lexèmes verbaux désignant des types de procès différents. L'imparfait tend à privilégier les procès statiques, appelés "états" (Il faisait beau), ou bien les procès dynamiques sans terme intrinsèque, appelés «activités » (Jean écrivait); selon les contextes, l'activité peut être présentée comme un procès «semelfactif » en train de se dérouler au moment considéré (Il écrivait, lorsque le téléphone sonna) ou bien comme une « itération » non bornée de procès identiques se répétant (Tous les matins, il écrivait). Le passé simple, de son côté, s'accommode mieux des procès dynamiques intrinsèquement bornés, appelés " achèvements » lorsqu'ils sont momentanés (Une bombe explosa) et " accomplissements » lorsqu'ils sont non momentanés (On construisit un immeuble).

Dans cet appariement entre temps verbaux et types de procès attachés aux lexèmes verbaux, le récit d'Echenoz présente (notamment dans les deux premières parties) un certain nombre de distorsions par rapport à l'usage canonique. D'où autant d'effets stylistiques, qui obligent le lecteur à un type particulier de travail interprétatif. Ces effets sont d'autant plus remarquables que, nous allons le voir, les distorsions surgissent au sein de configurations textuelles relativement stables, correspondant à des emplois canoniques dont l'interprétation locale se présente comme non problématique.

\subsubsection{Imparfait et type de procès}

Pour référer à des états ou à des itérations, certains imparfaits sont employés de façon classique avec des verbes appropriés, d'autres au contraire sont appariés de façon non canonique à des lexèmes verbaux.

Un exemple d'imparfait sur un verbe d'activité (normalement employé avec un sujet agentif) « recatégorisé » en état (du seul fait de la présence d'un sujet inanimé) a déjà été évoqué plus haut. D'autres exemples similaires figurent dans le texte: une cheminée de brique dont l'ombre donnait l'heure ( $\$ 1$ ), les vieilles constructions qui se collaient en grinçant 
contre lui [l'immeuble] (\$4), l'immeuble allait atteindre le ventre de sa mère (\$9), un terre-plein meuble prédisait le retour de la végétation trahie (\$11).

Tout à fait caractéristiques à cet égard sont les descriptions de l'image de la mère sur le mur, qui se prêtent, on le comprend aisément, à ce double jeu sur les traits " animé » / « inanimé »: elle les regardait de haut, tendait vers eux le flacon [...], elle souriait (§3), Sylvie Fabre luttait cependant contre son effacement personnel (\$8); plutôt que de «recatégorisation», c'est davantage d'«ambivalence» qu'il s'agit ici, puisque se superposent indissociablement l'évocation du personnage humain agent possible d'une activité, et l'état de l'image inanimée figeant la représentation de cette activité - d'où la perplexité du récepteur, à la première lecture.

Le lexème verbal est parfois compatible aussi bien avec un état qu'avec un processus. Certains emplois déroutants d'imparfaits avec des états se rencontrent alors, lorsque le contexte suggérerait plutôt des "achèvements " au passé simple. Ainsi, à la première phrase du paragraphe 1 (et donc du texte): Comme tout avait brûlé [...], c'était tout de suite beaucoup d'ouvrage, où la construction " comme + plus-que-parfait " et l'adverbe tout de suite font attendre ce fut beaucoup d'ouvrage, alors que l'imparfait induit une description statique qui ne fait pas avancer le récit, forçant le lecteur à se maintenir dans la situation initiale des deux personnages après l'incendie. De même, au début du paragraphe 7 : Négligence ou manouvre, on laissait l'espace dépérir est d'autant plus curieux que la suite enchaîne sur un aoriste (les choses vertes s'y raréfièrent) ; là encore, l'image d'une situation statique se trouve pour ainsi dire imposée.

Les emplois itératifs de l'imparfait sont assez nombreux dans la première partie du texte. Certains sont tout à fait classiques, comme ceux du paragraphe 2 , où l'interprétation itérative de l'imparfait est induite par la présence d'un circonstant initial de type générique: Le soir après le dîner, Fabre parlait à Paul de sa mère; il s'épuisait à vouloir la décrire; [...] soupirait Fabre; et le découragement l'endormait. Cette suite de procès itératifs se poursuit au début du paragraphe 3 , avec là encore un circonstant initial de type générique : Le dimanche et certains jeudis, ils partaient sur le quai de Valmy; ils allaient voir Sylvie Fabre; mais un décrochage interprétatif est nécessaire pour comprendre les imparfaits suivants comme des états (Elle les regardait de haut [...]). La suite de procès itératifs se retrouve encore au paragraphe $5:[. .$.$] s'énervait Fabre, que ce spectacle mettait en$ larmes; il pouvait aussi chercher la scène; l'effigie contre laquelle [...] rebondissaient ses reproches; dès qu'un attroupement menaçait de se former, mais cette suite intervient après une séquence de retours en arrière : Sylvie avait accepté de poser. Elle n'avait pas aimé cela. C'était trois ans avant la naissance de Paul - séquence elle-même suivie d'un état à l'imparfait qui assure le retour au repère temporel des procès itératifs: Paul, pour qui ce mur n'était qu'une tranche de vie antérieure.

L'interprétation itérative de l'imparfait n'est pas toujours évidente. En l'absence d'indication contextuelle univoque, le lecteur peut hésiter entre l'itération et l'activité semelfactive. Ainsi au paragraphe $8:$ Rassérénés, les chiens venaient compisser les planches; (ce que l'on devinait entre elles) faisait détourner le regard, où ces imparfaits interviennent après un plus-que-parfait marquant probablement un état résultant (elle [la palissade] s'était vite rompue). Sans doute est-ce là la description de procès itératifs (« tous les jours / chaque fois que Paul venait, voilà ce qu'il voyait ») plutôt que celle d'un procès unique en cours ("quand Paul revint, il vit que [...]»), mais la suite laisse la question ouverte en introduisant successivement un imparfait sur un état (Sylvie Fabre luttait) et un aoriste (Paul vit parfois [...]). Même hésitation possible au paragraphe 9, où la suite d'imparfaits: 
des hommes [...] la pelletaient; les planches [...] brûlaient; du ruban [...] balisait le théâtre est encadrée de part et d'autre par des aoristes : il y eut le trou / on lança ; trainèrent ; burent ; aperçut.

Dans d'autres cas, l'interprétation oscille entre un procès en situation et une vérité générale: Mais à partir des épaules, le chantier pour un fils devenait insupportable (\$10). Deux lectures semblent en effet entrer ici en conflit: d'une part l'actualisation (« une fois les épaules masquées, le chantier de construction était en train de devenir insupportable pour [le fils qu'était] Paul») et, de l'autre, la non-actualisation («quand les épaules [de l'image d'une mère] sont masquées, le chantier de construction devient un supplice pour un fils »).

Dans d'autres cas, enfin, l'imparfait supporte une pluralité possible d'interprétations, difficiles à démêler. Ainsi : (C'était un sépulcre au lieu d'une effigie de Sylvie,) on l'approchait d'un autre pas, d'une démarche moins souple $(\$ 10)$. Comment savoir ici si le groupe prépositionnel qualifie une itération de procès particuliers (la façon dont Paul et/ou son père approchait), une modalité déontique sur un procès générique (la façon dont tout un chacun devait approcher), ou bien encore un état - éventuellement modalisé - de l'objet (la façon dont le sépulcre était/ devait être approché)?

Dans la plupart de ces exemples, les incertitudes interprétatives correspondent à des cas d'ambivalence plutôt qu'à de véritables ambiguïtés, en sorte que le lecteur n'est pas obligé de choisir entre des solutions mutuellement exclusives, mais peut rester dans une relative indétermination, ou encore cumuler plusieurs lectures.

\subsubsection{Passé simple et type de procès}

Pour référer à des procès bornés, certains passés simples sont employés de façon classique avec des verbes sémantiquement appropriés, alors que d'autres sont, là encore, appariés à des types de lexèmes verbaux inattendus. De façon générale, le recours au passé simple permet de construire un procès de type "événement", dont la durée interne n'est pas prise en considération (valeur aoristique) et qui se présente comme totalement extérieur à tout point de vue subjectif (valeur de récit neutre et objectif). D'où un effet d'accélération du récit et d'inscription d'une sorte de trame historique, en rupture avec les tableaux descriptifs et itératifs créés par l'imparfait. Effet particulièrement frappant lorsque le lexème verbal se prête mal aux valeurs du passé simple.

En règle générale, le passé simple est plus facilement compatible avec un procès semelfactif qu'avec une itération, a fortiori lorsque le contexte induit des scènes itératives à l'aide d'imparfaits.

Tel est le cas, notamment, dans la première partie du texte. Par exemple, au paragraphe 2, qui décrit la vie quotidienne du père et du fils après l'incendie, la séquence d'imparfaits [...] Fabre parlait [...]; on ne possédait [...] ; il s'épuisait [...] est brusquement interrompue par la phrase au milieu de la cuisine naquirent des hologrammes que dégonflait la moindre imprécision; irruption étrange de ce passé simple, qui semble construire un événement marquant l'instauration objective et définitive d'hologrammes, ce que contredit immédiatement l'imparfait dégonflait - dès lors, pour résoudre ce conflit, le lecteur est conduit à reconstruire une série de "flashs événementiels » annulés aussitôt qu'instaurés. 

clôturée par un passé simple Souvent ce fut à Paul de déplier le canapé convertible qui rompt la série de scènes répétées (là où l'imparfait attendu c'était à Paul aurait au contraire maintenu de façon homogène cette série ouverte). mère [...] deux ou trois fois par mois ne pose pas de problème particulier, du fait du caractère borné du verbe visiter (au sens de « rendre visite à »), en revanche celle du paragraphe 8 : Paul vit parfois d'un ceil inquiet la pierre de taille chasser le bleu [...] est plus problématique, du fait du non-bornage intrinsèque du verbe de perception voir.

conflit possible entre la valeur du passé simple et celle du type de procès touche également les procès semelfactifs. Le récit des transformations successives des bâtiments entourant l'immeuble Wagner, dans la deuxième partie du texte, fait ainsi alterner appariements classiques et alliances détonantes.

Tout à fait canoniques sont les événements au passé simple construits à partir d'un inchoatif, d'un achèvement ou d'un accomplissement attribué à un sujet animé agentif (même aussi indéterminé que le on). Ainsi, au paragraphe $6:[.$.$] lorsqu'on se mit à démolir la$ vieille chose insalubre [...]; On remplaça la vieille chose par un bâtiment dynamique [...]; et, au paragraphe $9:[. .$.$] on lança la superstructure.$

En revanche, beaucoup plus inattendus sont ceux qui comportent un sujet inanimé. On a vu plus haut que des verbes au sémantisme intrinsèquement dynamique peuvent être compris comme des états lorsqu'ils ont un sujet inanimé et sont employés à l'imparfait : dans un tel cas, c'est le trait de dynamicité du verbe qui se trouve référentiellement gommé (même s'il continue, en quelque sorte, à être perçu en arrière-fond). Employés au passé simple, des verbes au sémantisme intrinsèquement dynamique vont au contraire conserver ce caractère et être compris comme des événements, ce qui tend à conférer le trait d'agentivité au sujet, même lorsqu'il est inanimé.

61 Tel est le cas au paragraphe $6:$ Le ravalement de la façade fit naître [...] une patine [...], au paragraphe $7:[. .$.$] une boue d'où saillirent des ferrailles aux arêtes menaçantes, ainsi qu'au$ paragraphe 9: de nouvelles planches neuves traînèrent un peu partout; Les étages burent Sylvie comme une marée. Dans un cas comme dans l'autre, le lecteur se trouve confronté à une tension conflictuelle dont la résolution ne conduit pas à une interprétation totalement univoque et stabilisée : il y a toujours surimposition de traits opposés.

L'un des plus beaux exemples d'emploi du passé simple est sans doute celui que l'on trouve au paragraphe 9 (il y eut le trou). Le paragraphe commence en effet sur une série de présents d'allure générique, de type vérité générale : Il suffit d'un objet pour enclencher une chaîne, il s'en trouve un toujours qui scelle ce qui le précède, colore ce qui va suivre [...]. Dès lors c'est très rapide, quelqu'un sans doute ayant vendu son âme avec l'espace, il y a le trou. Ce il y a le trou marque un état générique. Reprise par Il y eut le trou, tapissé de cette terre fraiche qui est sous les villes [...], la séquence se trouve transformée, sous l'effet du passé simple, en un événement unique dans une situation particulière: effet de surgissement du trou spécifique qui vient remplacer l'espace vert, prélude à la construction d'un nouvel immeuble.

63 Un dernier point mérite d'être signalé : temps du récit par excellence, le passé simple français est exprimé par des marques de conjugaison dont certaines sont homophones d'imparfaits du subjonctif. Ce n'est sans doute pas un hasard si, dans ce texte, certains imparfaits du subjonctif se trouvent employés dans un contexte de passés simples: Paul 
vit [...]; quoique tout cela restât très progressif (§ 8); Des semaines passèrent avant qu'il revînt quai de Valmy ( $\$ 10)$. Là encore, plusieurs formes semblent surimposées, qui laissent entendre simultanément une pluralité de lectures.

\section{Les présents de commentaire et leur insertion dans l'enchaînement narratif}

Dans L'Occupation des sols, comme dans la plupart des récits au passé, il arrive que l'on bascule du plan énonciatif qui supporte la narration (organisé du point de vue des temps verbaux autour de l'opposition imparfait/passé simple, et dans lequel l'énoncé se donne comme coupé de toute situation d'énonciation) à un plan énonciatif non narratif, dans lequel le narrateur manifeste sa présence pour effectuer des commentaires généraux sur les procès et états de choses évoqués dans le récit. C'est normalement un moment de suspension du récit, lequel fait place au «discours» du narrateur. Les temps verbaux constituent bien entendu un indice crucial de ce basculement puisque ces interventions du narrateur se font au présent. Ce présent, qui sert à asserter des vérités d'une portée générale, a alors une valeur omnitemporelle. En plus du présent, ces séquences textuelles de commentaire font intervenir des expressions à valeur générique (groupes nominaux, pronoms indéfinis, etc.).

Face à de tels décrochages énonciatifs, extrêmement courants, le récepteur attend simplement de trouver des indices du retour au plan énonciatif de la narration (en particulier les temps verbaux propres à ce plan), pour reprendre le récit exactement là où il l'avait quitté.

Dans le texte d'Echenoz, on trouve plusieurs passages qui présentent à première vue une telle utilisation du présent de commentaire. Mais ces passages au présent ne se contentent pas d'interrompre un moment le fil de la narration. Ils sont utilisés par Echenoz pour créer des effets de « changement de vitesse » dans la conduite du récit.

\subsection{Le présent de commentaire dissimule une progression du récit}

Dans la deuxième époque du récit, on trouve deux passages où le texte semble abandonner le plan énonciatif du récit historique pour des excursions discursives au présent. Ces passages se situent, dans la deuxième époque temporelle du récit, au moment où celui-ci traite de la transformation progressive de l'espace vert jouxtant l'immeuble Wagner, laquelle débouche sur le chantier de construction du nouvel immeuble.

\subsubsection{Une réflexion générale sur les réactions de l'usager?}

68 Le premier de ces passages se trouve au paragraphe 7. Les deux premières phrases $d u$ paragraphe portent en avant le récit en racontant le dépérissement de l'espace vert. La troisième phrase semble le suspendre pour faire place à un développement sur les réactions stéréotypiques des usagers face au dépérissement d'un espace vert :

Négligence ou manœuvre, on laissait l'espace dépérir. Les choses vertes s'y raréfièrent au profit de résidus bruns jonchant une boue d'où saillirent des ferrailles aux arêtes menaçantes, tendues vers l'usager comme les griffes mêmes du tétanos. [L'usager, volontiers, s'offense de ces pratiques. Heurté, l'usager boycotte 
cet espace rayé du monde chlorophyllien, n'y délègue plus sa descendance, n'y mène plus déféquer l'animal familier. Le trouvant un matin barré d'une palissade, il cautionne cette quarantaine l'œil sec, sans se questionner sur son initiative ; son cœur est froid, sa conscience pour soi.]

La palissade se dégraderait à terme : parfait support d'affiches et d'inscriptions contradictoires, elle s'était vite rompue à l'usure des choses, intégrée au laisseraller. conduit pas le récepteur à interpréter l'état de choses évoqué comme actualisé : on comprend «Si / Lorsque l'usager trouve cet espace barré d'une palissade, il cautionne 
cette quarantaine... » Jusqu'à la fin du paragraphe, l'utilisation du présent semble correspondre à une suspension du récit.

Pourtant, au paragraphe suivant, le texte débute par une phrase très déstabilisante: la palissade se dégraderait à terme : parfait support [...], elle s'était vite rompue à l'usure des choses [...].

77 En effet, cette phrase comporte à la fois le groupe nominal défini : la palissade et un verbe au conditionnel (qui exprime ici un procès dont la réalisation est envisagée comme postérieure à un repère passé). Le récepteur doit donc comprendre que l'on est revenu au récit, puisque le procès évoqué trouve son point de repérage temporel dans le passé. Le fait de coder cette instruction par un conditionnel demande au récepteur un effort interprétatif important. Plus déroutant encore, le GN défini : il suppose l'introduction préalable dans le récit d'un référent spécifique (puisque l'article défini code un référent supposé unique et connu du récepteur). Le récepteur a l'impression d'avoir « manqué un épisode »: ce référent, bien que codé par l'article défini comme connu, lui semble au contraire tomber de nulle part. Autant la suspension du récit était ménagée par une transition douce (via l'usager), autant sa reprise ne se fait guère en souplesse, le passage entre les deux plans énonciatifs est au contraire le lieu d'une accélération brutale, après un moment de (fausse) suspension. Il nous semble même que le lecteur est amené à relire le passage au présent.

78 En effet, bien que, en raison de l'utilisation du présent, ce passage se donne à lire comme un moment de suspension du récit, quelques détails, imperceptibles à première lecture, semblent maintenir une certaine ambiguïté.

79 Ainsi, alors que le démonstratif pluriel ces pratiques construit une référence nettement générique, la phrase suivante présente l'utilisation de cet espace. Le texte maintient le démonstratif mais revient au singulier: or, une perspective résolument généralisante aurait appelé le pluriel (L'usager boycotte ces espaces rayés du monde chlorophyllien). Bien entendu, c'est surtout le groupe participial Le trouvant un matin barré d'une palissade, qui permet une double lecture, grâce à l'ambivalence de l'article indéfini qui est interprété lors d'une première lecture comme référent à un élément quelconque, mais qui permet au récepteur, lors d'une (nécessaire) relecture, de trouver le référent spécifique que la phrase suivante suppose. L'article indéfini joue le même rôle pour le circonstant un matin, qui se lit une première fois comme un repère temporel quelconque (du type « un beau matin ») puis comme un repère spécifique.

Le récepteur doit donc relire la prédication secondaire véhiculée par le participe présent comme faisant en quelque sorte avancer le récit par contrebande, bien qu'elle soit intégrée à un passage discursif au présent. Tout semble fait d'ailleurs dans le texte pour qu'une double lecture soit possible: les présents de commentaire se donnent à lire comme génériques, mais le texte maintient une certaine ambiguïté, comme si le récit, bien qu'officiellement suspendu, continuait à avancer imperceptiblement, par-dessous, sans qu'on s'en rende bien compte. Echenoz semble chercher à redoubler, par la gestion du récit, ce que celui-ci raconte: la transformation de l'espace vert en chantier commence par un dépérissement, sorte de lente évolution quasiment hors du temps, puis s'accélère brutalement, sans que l'on ait vu arriver les choses: d'abord par l'apparition d'une palissade, ensuite, par celle du permis de construire. 


\subsubsection{Un événement du récit dissimulé dans un passage au présent ?}

81 Comme dans le passage précédent, le texte présente au début du paragraphe 9 un basculement énonciatif dans le plan du discours :

Paul vit parfois d'un œil inquiet la pierre de taille chasser le bleu, surgir nue, craquant une maille du vêtement maternel ; quoique tout cela restât très progressif. [Il suffit d'un objet pour enclencher une chaîne, il s'en trouve un toujours qui scelle ce qui le précède, colore ce qui va suivre - au pochoir, ainsi, l'avis du permis de construire. Dès lors c'est très rapide, quelqu'un sans doute ayant vendu son âme avec l'espace, il y a le trou]. Il y eut le trou, tapissé [...]

82 À nouveau, les verbes au présent se donnent à lire comme des présents de commentaire : ils marquent le basculement du texte dans un discours du narrateur, d'une portée extrêmement générale. Une différence importante avec le passage précédent est son mode d'insertion. Au paragraphe 7, l'excursion au présent hors du récit, se fait en douceur, grâce au GN «l'usager ", qui joue le rôle de cheville entre les deux plans énonciatifs. Par contre, la reprise du récit est extrêmement brutale, avec un effet d'accélération extrêmement déstabilisant.

83 Au paragraphe 9, on trouve quasiment la disposition inverse : le texte abandonne le plan énonciatif du récit très brutalement et le retrouve de manière plus liée, grâce à un effet de reprise ( ... il y a le trou. Il y eut le trou »). Lors d'une première lecture, on ne comprend pas très bien à quoi se rattache la première phrase du paragraphe 9 . En effet, l'ancrage référentiel de cette phrase au présent est extrêmement peu consistant: on se demande ce que dénotent exactement des expressions comme le GN indéfini «une chaîne" ou les relatives substantivées "ce qui le précède » et "ce qui va suivre ». A contrario, la phrase averbale qui suit (- au pochoir, ainsi, l'avis du permis de construire) ramène au contraire le lecteur dans l'univers du récit. Il n'en reste pas moins qu'elle prend sa valeur prédicative, par inférence contextuelle, de ce qui la précède, grâce à l'adverbe « ainsi ».

84 Par conséquent, elle est censée hériter de la valeur générique et omnitemporelle des présents de commentaire précédents. En toute logique textuelle, il faudrait donc gloser cette phrase averbale par: "ainsi, l'avis du permis de construire est un objet qui enclenche une chaîne, qui scelle ce qui le précède et colore ce qui va suivre ». Cependant, l'utilisation de l'article défini (dans un perspective généralisante on aurait plutôt dû avoir un indéfini) accompagné de la précision "au pochoir» (qui crée un "effet de réel» particularisant) sont deux éléments peu cohérents avec cette logique textuelle et amènent à une autre lecture.

Il faudrait alors comprendre que « l'avis du permis de construire, (réalisé) au pochoir, fut l'objet qui enclencha une chaîne, etc. ». Dans cette perspective, la première phrase de commentaire très généralisant porte sur un événement précis, actualisé dans le récit par la phrase averbale qui suit. On aurait donc, marqué typographiquement par le tiret, un retour éclair au récit, sous la forme d'une phrase averbale.

Il n'en reste pas moins que la phrase suivante est au présent: Dès lors c'est très rapide, quelqu'un sans doute ayant vendu son âme avec l'espace, il y a le trou. Pour le récepteur, ce maintien du présent code l'instruction que l'on n'a pas encore retrouvé le plan énonciatif du récit. Tout en se donnant à lire comme un passage de commentaire, ne faisant pas avancer le récit, le texte maintient une certaine ambiguïté. Ainsi, le circonstant initial de cette phrase (Dès lors) anaphorise un repère temporel précédemment installé, mais on ne 
sait pas très bien s'il s'agit du moment spécifique, dans le récit, de l'apparition de l'avis de permis de construire ou celui, quelconque, de l'apparition d'un objet qui enclenche une chaîne. En tout cas, cette phrase poursuit la réflexion sur la chaîne de causalité évoquée au début du passage, qui aboutit au trou. Pour autant, les verbes étant toujours au présent, les états de choses qu'elle dénote ne sont donc pas actualisés dans le récit, mais s'inscrivent dans une réflexion d'une portée très générale.

Par conséquent, bien que ménagée par un effet de soudure (il y a le trou. Il y eut le trou), la reprise du récit présente le même effet d'accélération qu'au paragraphe 8 . En effet, à l'issue de la réflexion très générale menée lors du passage au présent, le récit reprend à partir d'un état de choses plus avancé que celui où il s'était arrêté. Une étape, celle du permis de construire, a été glissée dans le passage au présent. Tout se passe donc comme si le passage au présent faisait avancer, par-dessous, le récit.

\subsection{Le présent de commentaire ménage une transition vers un récit qui se fige progressivement sur une image}

La toute fin du texte présente une utilisation remarquable du présent de commentaire :

Ils mangèrent donc vers quatorze heures mais sans grand appétit, leurs mâchoires broyaient la durée, la mastication n'était qu'horlogère. [D'un tel compte à rebours on peut, avant terme, convoquer à son gré le zéro. Alors autant s'y mettre, autant gratter tout de suite, pas besoin de se changer, on a revêtu dès le matin ces larges tenues blanches pailletées de vieille peinture, on gratte et des stratus de plâtre se suspendent au soleil, piquetant les fronts, les cafés oubliés. On gratte, on gratte et puis très vite on respire mal, on sue, il commence à faire terriblement chaud.]

On constate que dans ce passage au présent, la première phrase (D'un tel compte à rebours on peut, avant terme, convoquer à son gré le zéro) présente tous les indices d'un présent de commentaire: d'une part, une expression anaphorique (un tel compte à rebours) qui résume le contenu de la phrase précédente, tout en construisant une référence quelconque, et d'autre part, le pronom indéfini « on » qui a ici une valeur proprement générique. Cette première phrase semble servir à ménager une transition entre deux régimes de récit. On passe en effet d'un régime de récit " traditionnel » où les événements sont dénotés par des verbes au passé simple à un régime paradoxal où on ne trouve plus que du présent. Encore une fois, le présent de commentaire est utilisé pour gérer un changement de vitesse du récit.

La reprise du récit, sans qu'elle ait véritablement lieu, est impliquée par les deux phrases averbales: Alors autant s'y mettre, autant gratter tout de suite, dont le statut est difficile à définir. Ces deux phrases semblent mêler les effets de voix: on peut y entendre les paroles des personnages (« autant s'y mettre, autant gratter tout de suite ») rapportées au discours indirect libre, mais aussi la voix du narrateur qui annonce une nouvelle étape dans le processus raconté par le récit. Toutefois, il est certain que le lecteur est amené à interpréter ces deux phrases comme signalant l'accès à une nouvelle étape dans le récit : les deux personnages commencent leur entreprise de grattage.

91 Cette reprise du récit se fait cependant de manière paradoxale : les phrases qui suivent ne rapportent pas d'événement mais décrivent plutôt des états. En effet, le passé composé « on a revêtu » a strictement une valeur de présent accompli (mutatis mutandis, il équivaut à « on porte ») et décrit donc simplement un état résultant. La phrase qui suit (on gratte) ne pose pas un événement qui ferait en lui-même avancer le récit : associé au présent, 
l'aspect imperfectif du procès "gratter » donne à voir une activité stable, sans limite finale, seulement animée du dynamisme interne et périodique du procès. Du point de vue de l'aspect, la phrase suivante (des stratus de plâtre se suspendent au soleil) présente les mêmes caractéristiques : l'aspect lexical imperfectif associé au présent traduit un état de choses bien plutôt qu'un événement.

La fin du texte se poursuit selon le même principe, puisqu'elle associe des procès à l'aspect imperfectif (gratter, respirer, suer) au présent, dont l'aspect grammatical ne suppose aucune limite, ni finale, ni initiale. Enfin, la dernière phrase du texte (il commence à faire terriblement chaud), qui fait intervenir l'aspect inchoatif, clôt le récit de manière paradoxale. Elle présente le dernier procès immédiatement à son début, en laissant dans l'ombre, pour ainsi dire, l'essentiel de son déroulement. Ce procédé clôt brutalement le récit après une série d'images arrêtées, sans que soit rapportée une véritable succession d'événements.

93 En effet, dans ce dernier passage au présent, c'est à nouveau au lecteur qu'il revient de reconstituer le récit, en mettant en rapport les états résultants d'événements qui ne sont pas exprimés en tant que tels. L'utilisation du présent, appliqué à des procès imperfectifs produit l'impression que les états de choses évoqués se superposent, comme des images en surimpression, au lieu d'être enchaînés dans une succession chronologique.

En somme, le lecteur est amené à réinjecter de la temporalité entre les images fixes, afin de retisser la trame du récit qui produit, de manière sous-jacente, ces images. Ainsi, entre (Alors autant s'y mettre, autant gratter tout de suite) et (on gratte), le lecteur doit supposer que les personnages se sont mis à l'ouvrage. De la même manière, le lecteur est amené à mettre en rapport (on gratte) et (et des stratus de plâtre se suspendent [...]) et à comprendre que le dernier état résulte du premier, donc lui succède. Le texte lui fournit tout de même quelques appuis, sous la forme de circonstants : tout de suite, au début donne le signal de la reprise du récit, c'est-à-dire du début de l'entreprise de grattage, et puis très vite, dans la dernière phrase, soutient la nécessaire successivité chronologique. De la même manière, le connecteur et (entre on gratte et des stratus se suspendent [...]) suggère légèrement une relation de cause à effet (donc de successivité chronologique). Autre effet, la répétition de on gratte, on gratte exprime la durée du processus et réinjecte de la durée dans cette série d'images fixes.

Il faut également souligner que cette fin du récit est rendue extrêmement floue par l'escamotage des participants. En effet, après la phrase au présent de commentaire (D'un tel compte à rebours on peut [...]) qui présente l'utilisation du pronom indéfini on, le texte maintient ce pronom comme sujet des verbes conjugués au présent. Toutefois, il s'agit d'une fausse continuité thématique puisque dans la première phrase au présent le pronom on a une valeur clairement générique, tandis que dans la suite du texte, le lecteur, invité à reconstruire le récit, doit comprendre qu'il s'agit de Fabre et de Paul.

On trouve par ailleurs un indice qui empêche de relire toute la fin du texte comme un développement générique sur ce type de situation et la rattache au récit : il s'agit de la mention du soleil, auquel se suspendent les stratus de plâtre. Ce détail avait en effet été mis en avant à deux reprises par le récit au début du paragraphe 15 (Le soleil en effet balaierait [...] et Un soleil comme celui-ci, développa [...]).

En somme, l'usage du présent à la fin du récit crée un effet de ralentissement qui donne à voir les derniers procès comme une série d'images fixes qui se surimposent, sans véritablement être enchaînées narrativement. L'aspect inchoatif appliqué au dernier 
verbe donne l'impression d'un arrêt sur image arbitraire, comme si le mouvement de ralentissement engagé plus tôt était arrivé à son terme.

\subsection{Les interventions subreptices du narrateur}

Les autres occurrences de présent correspondent à des microruptures de plan énonciatif. Pour autant, elles n'ont aucune incidence dans l'enchaînement narratif: le récit est comme suspendu un court instant pour faire place à une intervention du narrateur. À la limite, il semble qu'elles aient pour seule fonction de permettre au narrateur de manifester sa présence, dans une sorte de clin d'œil au lecteur averti, afin de pointer subrepticement l'artificialité du système énonciatif propre au récit historique, selon laquelle l'énoncé se donne comme coupé de la situation d'énonciation.

Plus tard, suffisamment séparé de Fabre pour qu'on ne se parlât même plus, Paul visita sa mère sur un rythme plus souple, deux ou trois fois par mois, compte non tenu des aléas qui font qu'on passe par là.

Cette utilisation du présent est difficile à expliquer. La logique du récit aurait appelé l'imparfait et le pronom il (compte non tenu des aléas qui faisaient qu'il passait par là). De plus, l'emploi du pronom indéfini on invite à une interprétation générique qui est difficile à concilier avec la valeur spécifique de l'adverbe là (qui renvoie anaphoriquement à l'endroit où se trouve la mère de Paul). Au total, cette séquence semble relever autant du commentaire (le pronom on et le présent) que du récit (l'adverbe là) sans qu'il soit possible de départager clairement les deux plans énonciatifs.

Paul, dont c'est quand même le nom, se tourna vers celle qui criait Fabre Fabre encore, j'ai du lait.

Ici, l'incidente vient expliquer pourquoi Paul se retourne au nom de Fabre, qu'il partage avec son père. Seulement, on aurait plutôt attendu un imparfait, qui aurait intégré cette explication dans le plan énonciatif du récit. L'utilisation du présent donne l'impression que le narrateur s'adresse un instant au lecteur pour jeter, du dehors, un coup d'œil sur le récit. Coup d'œil qui semble signifier : il ne vous a pas échappé que mes personnages, bien qu'entretenant des relations difficiles, sont tout de même père et fils et portent par conséquent le même nom de famille.

La femme s'éloignait, on ne sait pas qui c'était.

Cette utilisation du présent est comparable à la précédente : le présent traduit une prise de parole du narrateur qui se manifeste en tant que tel. Toutefois, cette occurrence est franchement troublante car ici le narrateur intervient pour exprimer un défaut d'information sur un élément du récit. Le narrateur, en même temps qu'il dévoile sa présence, en arrière-plan du récit, adopte la posture d'un simple témoin de la scène. D'ailleurs, l'utilisation du pronom « on » renforce cet effacement paradoxal du narrateur : en effet, le pronom indéfini on peut aussi bien renvoyer au narrateur, qu'au lecteur, qu'aux deux instances, prises ensemble et considérées un moment côte à côte en train de suivre le récit.

Ils s'exprimèrent également peu sur la difficulté de leur tâche qui requerrait, c'est

vrai, de la patience et du muscle, puis des scrupules d'égyptologue en dernier lieu.

Il est possible de voir dans cette phrase un effet de voix. La phrase évoque en effet une prise de parole des personnages (Ils s'exprimèrent). Dans cette optique, on peut voir dans la relative une manière de rapporter au discours indirect libre les paroles des personnages. L'incidente au présent correspond alors à une intrusion du narrateur, qui vient donner 
son approbation - au discours direct - aux paroles des personnages - rapportées au discours indirect libre. Le procédé paraît tout à fait cocasse : d'une part, le narrateur se pose en témoin du récit, qui donne son avis en passant, d'autre part le procédé confronte une manière très délicate de rapporter les paroles des personnages à une intrusion brutale du narrateur. Cependant, comme s'il s'agissait d'augmenter encore un peu l'insécurité du lecteur, cette interprétation est rendue incertaine par l'utilisation de l'adverbe peu qui limite la représentation d'une prise de parole des personnages et perturbe par conséquent le fonctionnement du discours indirect libre.

\section{Les conditionnels et leur mode d'insertion dans la trame événementielle}

On trouve dans L'Occupation des sols six occurrences de conditionnel. Notons d'emblée que ces conditionnels ne situent pas les procès concernés dans un monde hypothétique, mais bien dans le monde « réel » du récit. En effet, les six occurrences du texte ne sont pas construites en corrélation avec l'expression d'une hypothèse.

Les conditionnels du texte ont au contraire une valeur temporelle et contribuent, bien qu'étrangement, à faire avancer le récit. Leur valeur est prospective : ils projettent des procès et des états de choses depuis les époques du récit (situées dans le passé) vers une époque ultérieure. Toutefois, pour prendre cette valeur temporelle, le conditionnel doit normalement être relié à une indication explicite du passé (du type je savais que cela arriverait), ce qui n'est jamais le cas dans le texte d'Echenoz.

L'autre utilisation prototypique $\mathrm{du}$ conditionnel prospectif correspond au fait de transposer dans le discours indirect libre, le futur employé au discours direct. Malheureusement, le texte ne permet pas de rattacher clairement nos occurrences de conditionnels à des prises de paroles des personnages. Étant donné ces éléments, l'utilisation du conditionnel est particulièrement insécurisante pour le lecteur qui ne sait trop comment l'interpréter. Il n'en reste pas moins que celui-ci est amené à tenir compte de ces conditionnels lors du travail de recomposition de la trame événementielle que lui demande le texte. On peut rassembler ces six occurrences de conditionnel en deux groupes : les trois premières ont pour point d'appui la seconde époque du récit, les trois suivantes la troisième et dernière époque du récit.

\subsection{Les conditionnels de la seconde époque du récit}

Le premier conditionnel, extrêmement déstabilisant, se rencontre au tout début du paragraphe 8: La palissade se dégraderait à terme. Sa valeur prospective est assurée par l'emploi du circonstant à terme. Ce conditionnel joue un rôle capital dans la gestion du récit, puisqu'il donne le signal que le texte revient au plan énonciatif du récit, après une réflexion générale ( $\mathrm{du}$ moins en apparence) sur les réactions de «l'usager » face au dépérissement des espaces verts. En plus de cette information, cette phrase apprend au lecteur qu'au point où le récit reprend, une palissade est apparue autour de l'espace vert, marquant ainsi une nouvelle étape dans le récit, et lui demande en même temps d'envisager un procès situé au-delà de ce repère. En somme, l'insertion de ce conditionnel dans la trame des événements du récit produit un brutal effet d'accélération dans la conduite du récit. 
107 Par ailleurs, comme la valeur prospective consiste à considérer du point de vue d'une époque passée la réalisation d'un procès dans un futur, notre conditionnel implique « un point de vue» implanté dans l'époque passée en question. Malheureusement aucun « point de vue » du type « On savait que la palissade se dégraderait à terme » n'est indiqué ni même susceptible d'être reconstruit par inférence contextuelle. Il n'en reste pas moins que le conditionnel donne l'impression que l'évolution du récit est annoncée de manière prophétique par une instance difficile à définir.

Les deux occurrences suivantes correspondent aux deux premières phrases du paragraphe 10. On trouve à nouveau des indices forts de la valeur prospective de ces conditionnels (les circonstants «Au lieu de l'espace vert» et "plus tard»). Il est intéressant de noter que l'on trouve à nouveau ces conditionnels en début de paragraphe et qu'ils produisent un effet comparable d'accélération du récit. Alors que le paragraphe 9 se termine au moment où l'immeuble est en construction, les deux conditionnels projettent le récit vers la description d'une construction achevée. Le futur qu'ils visent, tout à fait indéterminé (plus tard), est en outre susceptible d'aller au-delà de la troisième époque du récit, au moment de laquelle "l'édifice [n'est] pas entièrement achevé ». Par ailleurs, la phrase suivante revient à une étape antérieure (à partir des épaules). Les deux conditionnels viennent donc s'insérer étrangement dans la trame des événements : ils dénotent un état de choses qui oblige le lecteur à se propulser en avant, mais une fois ce «bond" effectué, il lui faut revenir en arrière et reprendre le fil "normal» des événements.

\subsection{Les conditionnels de la troisième époque du récit}

Les trois autres occurrences de conditionnel se rencontrent au début du dernier paragraphe. On note qu'à nouveau, le conditionnel ouvre un paragraphe, projetant les procès qui constituent le récit dans une époque future.

Le soleil en effet balaierait tout le studio, comme un projecteur de poursuite dans un music-hall frontalier. [...] Ainsi que tous les jours chômés, les heures des repas tendraient à se glisser les unes sur les autres, on s'entendit pour quatorze heures - ensuite on s'y met. [...] Ils s'exprimèrent également peu sur la difficulté de leur tâche, qui requerrait, c'est vrai, de la patience et du muscle, puis des scrupules d'égyptologue en dernier lieu.

110 Le premier conditionnel ouvre une période qui va jusqu'à la fin de la journée du dimanche. Sa valeur prospective est comparable à celle des occurrences précédentes, avec à nouveau le problème de la détermination de l'instance énonciative sous-jacente. En effet, l'emploi du conditionnel serait canonique si la phrase était enchâssée dans une phrase de type « Fabre expliqua à Paul que le soleil balaierait tout le studio. » L'hypothèse $\mathrm{du}$ discours indirect libre peut éventuellement être sauvée, dans la mesure où le paragraphe précédent se termine par des paroles rapportées au discours direct. Néanmoins, le passage du discours direct au discours indirect libre, à l'occasion d'un changement de paragraphe paraît extrêmement difficile à suivre. Il semble plutôt que ce soit ici une instance énonciative du côté du narrateur qui réagisse aux propos de Fabre, pour surenchérir, ce qui expliquerait l'emploi de en effet.

Les deux dernières occurrences de conditionnel semblent avoir la même valeur prospective que les précédentes. Néanmoins, la proximité de verbes de parole brouille un peu cette valeur en laissant entendre des échos de discours indirect libre. Ainsi, la 
seconde occurrence précède immédiatement le verbe de parole on s'entendit pour quatorze heures. L'emploi du conditionnel pourrait servir à rapporter le raisonnement d'un des personnages, qui conduit à la décision de fixer une heure. Cette hypothèse est tout de même difficile à maintenir, étant donné que le verbe de parole au passé simple vient après le conditionnel.

La dernière occurrence semble offrir le plus de crédibilité à un usage du conditionnel visant à rapporter indirectement les paroles des personnages. On trouve en effet le verbe "s'exprimer ", ainsi que l'étrange intervention du narrateur (c'est vrai). Toutefois, cette interprétation n'est pas la seule possible et on peut aussi lire dans ce conditionnel une valeur prospective.

\section{Les participes et leur contribution à l'histoire}

113 Il reste à dire quelques mots des participes et de leur contribution au récit. Contribution assurément modeste. Les participes sont bien les formes «ancillaires» que décrit la grammaire, serviteurs obscurs et sans grade, peu visibles à la première (et même à la seconde) lecture, formes hybrides, participant (comme leur nom l'indique) à la fois du verbe, par leurs propriétés aspectuelles et rectionnelles, et de l'adjectif, par leur dépendance et leur rattachement nécessaire à un support nominal (contrastant avec la fière indépendance et le statut d'égalité d'un verbe à mode personnel par rapport à son sujet), - et complètement inaptes par elles-mêmes à inscrire un procès dans le temps. Ce ne peut donc être que secondairement, de façon indirecte, que de telles formes peuvent enrichir ou infléchir une narration, selon le détail de leur emploi et les inférences auxquelles celui-ci invite. Mais en même temps ce statut de "second rôle » ouvre des possibilités d'utilisation ambiguë, ou en demi-teinte, dont le texte ne manque pas de jouer subtilement. Nous en distinguerons deux grands types.

\subsection{Les participes adjectivaux, entre propriété et fragment d'histoire}

114 Le premier type concerne des emplois dans lequel le participe se rattache étroitement à un nom; il est alors épithète, et la plupart du temps il suit son $\mathrm{N}$ tête sans en être séparé par une virgule (bien que ce critère ne soit pas absolu). Cette configuration indique que le participe est en emploi essentiellement adjectival. Sa valeur verbale (valeur de procès, valeur prédicative) est alors a priori complètement, ou quasi complètement, estompée, il ne marque plus qu'une simple propriété attachée statiquement au référent dénoté par un nom, fondue en quelque sorte dans l'immanence de l'image portée par le nom, - quelle que soit par ailleurs la façon dont cette image intervient dans l'histoire (comme participant d'un événement, d'une description...).

Dans le cas du participe passé, cette propriété est le fruit de son aspect accompli, porté jusqu'au stade d'une propriété résultante intégrée: calques millimétrés, piquets rouillés, regard mort, nuances insoupçonnées, balconnets incurvés, etc. Dans le cas du participe présent, elle est le fruit de son aspect en accomplissement combiné à la valeur imperfective du verbe (les verbes perfectifs étant exclus de ce fonctionnement), menant là encore à une propriété caractérisant un référent à la manière d'un adjectif (comme en témoignent l'accord avec le nom support, et l'étiquette grammaticale d' «adjectif verbal ») : anneaux gluants, voix énervante, arêtes menaçantes (aussi, nonobstant la virgule et le caractère régissant du participe : une mission supérieure, relevant d'une cause...). 
116 Mais dans plusieurs occurrences la propriété rattachée à un référent, si elle se donne à lire comme une propriété adjectivale, n'en suppose pas moins une émergence qui la rattache à l'histoire, pouvant conduire en quelque sorte à une lecture double, ambivalente, conservant une part de prédicativité du participe : dans les planches brisées de la palissade brûlaient sans flamme, le participe brisées marque à la fois une propriété descriptive (les planches "en morceaux») et le processus qui y a conduit (les planches ont été brisées). La double lecture se fait ici sans difficulté, mais il n'en va pas toujours de même.

Ainsi la cour dallée que traverse celui (Paul ?) qui entre dans le nouvel immeuble s'impose en bloc comme une notation descriptive, perception anhistorique, d'un seul coup, mais elle n'en suppose et/ou n'en déclenche pas moins un travail d'interprétation, reconstituable au compte de celui qui entre (et, in fine, chez le lecteur) menant à la prise de conscience de l'effectuation du dallage, qui s'inscrit dans la série des opérations de construction du nouvel immeuble. Le participe "écrase » pour ainsi dire le processus et son résultat. On ne sait plus alors exactement de quel point de vue relève le texte, compromis entre un compte rendu de perception brut (mais de qui ?), et la reconstitution (mais par qui ?) d'un processus. Dans la suite de la même phrase du texte (un terre-plein meuble prédisait le retour de la végétation trahie), une interprétation stricte du participe trahie serait sans doute hasardeuse, entre une propriété (fortement teintée d'affectivité) d'un référent, au demeurant virtuel, à venir, et le rappel de faits révolus.

Cette double valeur (propriété d'un référent / trace d'un événement ou d'un moment de l'histoire) se retrouve fréquemment dans des participes épithètes (gardes $d u$ corps $d u$ Wagner préservé). L'inscription narrative peut être renforcée par la présence d'éléments régis par le participe, ou soulignée par des adverbes comme encore, déjà : les planches déjà gorgées de colle et d'encre, promptement corrompues, - ou encore rester implicite (le sol inachevé, les cafés oubliés), à charge au lecteur, dans chaque emploi, de réaliser sa pondération personnelle, de modeler en quelque sorte à sa façon le compte rendu de l'histoire, en réglant le rapport entre la chronologie des faits et le (ou les) temps de la découverte ou de l'interprétation.

\subsection{Les participes prédicatifs, et leur insertion narrative}

Le second type d'exploitation des participes pour l'embellissement de la narration concerne les participes mis en relief, et dans une certaine mesure autonomisés, par détachement, en début de phrase, ou dans la partie finale, derrière le verbe. Par-delà leur rattachement toujours nécessaire à un nom (sauf cas particulier, le sujet de la phrase), c'est leur valeur prédicative (et rectionnelle) qui prend le dessus, et c'est leur rapport au verbe principal qui devient alors crucial pour l'interprétation. À cet égard, il n'y a pas lieu de faire une différence entre les participes (ou groupes participiaux) ordinaires, et les participes dotés de ce qui ressemble à un sujet propre, constituant une "proposition participiale " (Paul considérant cela, une femme s'arrêta... ; Les fondations enracinées, ..., on lança ...), qui posent les mêmes problèmes de rapport au verbe principal. Ce rapport (de correspondance régulière dans les cas standard) n'est pas exempt de marges d'interprétation; de surcroît, il peut être diversifié et devenir source d'effets marqués dans d'autres configurations.

Le cas standard se rencontre dans des exemples tels que :

- Sylvie Fabre luttait, [...], bravant l'érosion éolienne ( = « elle bravait [...]») 
- des hommes [...] la [ = la terre] pelletaient [...], s'aidant de machines ( = « ils s'aidaient [...]»)

- les vieilles constructions qui se collaient [...] contre lui, terrifiées par [...] ( = « elles étaient terrifiées $[. .] »$.

- il dormait contre le sourire, suspendu [...] ( = « il était suspendu [...]»)

121 Le participe (relevant généralement du même type de procès que le verbe principal) s'interprète comme aligné temporellement et aspectuellement sur ce verbe: concomitance temporelle + identité aspectuelle. Si le verbe lui-même a un statut clair au regard de la progression narrative, comme dans les exemples ci-dessus à l'imparfait descriptif, le participe est alors pleinement dans ses fonctions ancillaires. Son effet textuel de base consiste simplement à assigner un statut de second rang au procès qu'il dénote (et à ses actants propres éventuels), par rapport au procès du verbe de référence, avec son cortège actanciel.

Il peut en être de même avec d'autres temps que l'imparfait (Plus tard tous deux seraient solidaires, projetant leur ombre protectrice $[. .]=$. «ils projetteraient $[. .] »$.$) , ainsi que dans les$ emplois du gérondif avec en ([...] soupirait Fabre en posant une main [...]; les vieilles constructions qui se collaient en grinçant [...]; le veuf parlait [...] en dépliant [...]).

Mais en même temps, c'est le caractère relativement peu spécifié, indéterminé, de ce rapport qui est susceptible de susciter des problèmes d'interprétation: quel lien (référentiel, logique) faut-il établir entre les procès ainsi placés à des niveaux différents, sans que ce qui les unit ou les différencie soit explicité ? La hiérarchie est explicitement posée, mais non motivée, ce qui pousse plus ou moins clairement le lecteur à en chercher les raisons, - qui, bien entendu, sont diverses et subjectivement appréciées. $\mathrm{Si}$, a fortiori, le rapport temporel/aspectuel que le participe entretient avec le verbe principal n'est pas strictement standard, ou si le verbe principal lui-même est sujet à des problèmes d'interprétation - l'un des cas n'excluant pas l'autre -, le participe peut devenir source d'incertitude ou d'ambiguïté de la lecture.

La position du participe à l'initiale, en préfixe au noyau de la phrase, est particulièrement de nature à susciter des effets particuliers de lecture, concernant le rapport temporel et/ ou sémantique entre les procès. Dans la phrase : disjointes, ce que l'on devinait entre elles [les planches de la palissade] faisait détourner le regard, le participe à l'initiale, s'il se comprend immédiatement, au fil de la lecture enchaînant sur la phrase précédente après les deux points, comme se rapportant aux planches, oblige néanmoins à un enchaînement complexe, syntaxiquement (l'enchaînement ne se fait pas sur le sujet), et sémantiquement: le lecteur doit reconstituer une relation à la fois chronologique et causale (les planches sont devenues disjointes, ce qui permet de voir à travers les interstices). Économie et concentration d'effets s'obtiennent ici au prix d'une construction que seul le participe rend possible.

Dans la phrase : mastiquées depuis peu, les vitres étaient encore barrées [...], on retrouve dans le participe l'association d'une valeur de propriété et d'une valeur d'inscription de fait, d'où une hésitation possible sur le temps qui serait utilisé dans une paraphrase : « comme les vitres étaient / avaient été mastiquées depuis peu [...]».

Les emplois dans des phrases au présent sont, on l'a vu ci-dessus, particulièrement troubles: le participe prolonge et amplifie les incertitudes de la lecture; ainsi dans Le trouvant un matin barré d'une palissade, il [ = l'usager] cautionne cette quarantaine.

Le participe apparaît bien, en définitive, comme une ressource d'appoint, ajoutant son lot tant de précisions que de petites énigmes, qui apportent à la construction narrative une 
série de contributions souvent subreptices. Sans jamais mettre en danger l'interprétation sur un point crucial, les participes donnent au lecteur une certaine marge de lecture et contribuent ainsi à lui conférer un rôle de constructeur actif du sens du texte.

\section{Conclusion}

$\mathrm{Au}$ total, les temps verbaux apparaissent comme des éléments cruciaux de la construction du récit : leur emploi est en bonne partie responsable de l'originalité et de la saveur du texte de L'Occupation des sols. Sources a priori de la cohérence chronologique de l'histoire reposant sur l'enchaînement des événements, ils peuvent également déstabiliser la lecture et obliger le récepteur à des efforts de reconstruction inédits produisant une cohérence plus riche, plus inattendue, susceptible de conserver des zones problématiques. C'est tout le plaisir de la lecture, c'est tout l'art de l'écriture, pour qui sait jouer en virtuose des ressources de la langue, comme sait le faire Echenoz.

\section{RÉSUMÉS}

L'emploi des temps verbaux dans L'Occupation des sols ne s'inscrit que partiellement dans le jeu narratif classique fait de complémentarité entre passé simple (support d'événements) et imparfait. Le lecteur doit en plus, pour restituer la cohérence temporelle de la narration, lutter non seulement avec des retours en arrière, des non-dits et des «trous » chronologiques, avec des imparfaits ou des participes ambivalents, mais aussi avec certains passés simples déroutants (incongrus ou non intégrables dans une successivité), et avec des présents et des conditionnels qui sous leur première apparence de commentaires du narrateur, sont nécessaires à la progression du récit. Parfois déstabilisé, mais pris au jeu, le lecteur doit participer à la construction de la cohérence : plaisir de la lecture, art de l'écriture.

The use of tenses in L'Occupation des sols only partially matches the usual narrative pattern, whereby the 'passé simple' (for events) and the 'imparfait' jointly build a coherent story. In order to ensure the temporal coherence of L'Occupation, the reader must make his way non only through flashbacks, missing elements, or chronological 'gaps', not only through ambiguous 'imparfaits' or gerunds, but also through a few misleading 'passés simples' (unexpected, or not fit for chronological succession), and through 'présents' and 'conditionnels' which, though purely commentative at first sight, prove to be necessary to the narration's progress. The reader may sometimes be abashed, but he is also captured, and plays an active role in building coherence : reader's delight, writer's art.

\section{INDEX}

Mots-clés : temps verbaux, cohérence temporelle, narration, lecture

Keywords : tenses, temporal coherence, reading 


\section{AUTEURS}

\section{CATHERINE FUCHS}

Directeur de recherches émérite, laboratoire Lattice CNRS-ENS-Université Paris 3.

Parmi les publications :

La Paraphrase, Paris, PUF, « Linguistique Nouvelle », 1982.

Paraphrase et énonciation, Paris, Ophrys, « L'Homme dans la Langue », 1994.

Les Ambiguïtés du français, Paris, Ophrys, « L'Essentiel Français », 1996.

B. Victorri et C. Fuchs, La Polysémie : construction dynamique du sens, Paris, Hermès, 1996.

L. Florea et C. Fuchs, avec la collaboration de F. Mélanie-Becquet, Dictionnaire des verbes du français actuel : constructions, emplois, synonymes, Paris, Ophrys, « L'Essentiel Français », 2010.

La Comparaison et son expression en français, Paris, Ophrys, « L'Essentiel Français », 2014.

\section{PIERRE LE GOFFIC}

Professeur émérite, université Paris 3, laboratoire Lattice CNRS-ENS-Université Paris 3.

Parmi les publications :

«Formes en -ant et contexte », in C. Guimier (éd.), Co-texte et calcul du sens, Presses universitaires de Caen, 1997, p. 123-133.

Les Formes conjuguées du verbe français oral et écrit, Paris, Ophrys, 1997.

Grammaire de la phrase française, Paris, Hachette, 2004 (1993).

"La phrase "revisitée" ", Le français aujourd'hui, 148, 2005, p. 55-64.

«Phrase, séquence, période », in D. Van Raemdonck (éd.), Modèles Syntaxiques. La syntaxe à l'aube du XXI siècle, Berne, Peter Lang, 2008, p. 329-356.

"Phrase et intégration textuelle », Langue française, 170, 2011, p. 11-28.

\section{MATHIEU MAILLARD}

Étudiant M2 en sciences du langage, université Paris 3. 